\title{
A Case Report of Severe Minocycline-Induced DRESS Affecting Multiple
}

\section{Organs}

\section{Marte Kase | Bartlomiej Witczak | Willy Aasebø*}

*Correspondence: Willy Aasebø

Address: Section of nephrology, medical department, Akershus University Hospital, Norway

e-mail $\bowtie:$ wiaa@ahus.no

Received: 25 April 2020; Accepted: 04 May 2020

Copyright: (C) 2020 Kase M. This is an open-access article distributed under the terms of the Creative Commons Attribution License, which permits unrestricted use, distribution, and reproduction in any medium, provided that the original work is properly cited.

\section{ABSTRACT}

Drug Reaction with Eosinophilia and Systemic Symptoms (DRESS) may affect multiple organs, and may be fatal. This case illustrates the seriousness of the syndrome and that several different organs systems may be involved. In the reported patient, the following organs were involved during the illness: skin, kidneys, lungs and lymph glands. However, CNS, red blood cells, thrombocytes and electrolytes may also have been directly or indirectly involved in the syndrome. The patient was very ill and his kidney function never recovered.

Keywords: Allergy, Eosinophil Erythrocytes, DRESS, Kidney Failure

\section{Introduction}

Drug Reaction with Eosinophilia and Systemic Symptoms (DRESS) is a rare and potentially lifethreating syndrome. This condition may affect multiple organs; most commonly skin, blood (eosinophilia, atypical lymphocytosis), lymph glands and internal organs (liver and lungs) (Husain et al., 2013a; Husain et al., 2013b). The symptoms usually start two to eight weeks after drug exposure, and recurrent relapses are common despite discontinuation of the culprit drug.

We report a case of severe minocycline-induced DRESS syndrome. The patient was admitted to hospital with a severely itching, macular rash and acute kidney injury. He developed multi organ affection, additionally he developed severe hypercalcemia, encephalopathy, hemolytic anemia and thrombocytopenia, which never have been reported previously related to DRESS.

\section{Case Report}

From previously, the 79 year old male patient had done Y-graft surgery in 2010 due atherosclerosis. He had chronic kidney disease stage 3, probably caused by nephrosclerosis. In 2015 the Y-graft became chronically infected with Propionebacterium acne. Antibiotic therapy was difficult due to 
allergic reactions to different antibiotic agents. Two weeks before admittance he started with Minocycline and was admitted with a severely itching generalized confluent macular rash and acute on chronic kidney failure with elevation of serum creatinine from 150 to $400 \mu \mathrm{mol} / \mathrm{L}$. Results of initial blood samples were Hb: 8.3 g/dl, MCV: 94 (fL), thrombocytes: 58 x109/L, leucocytes: 22.9 x 109/L, of which 12.8 x 109/L were eosinophilic leucocytes, C-reactive protein: $50 \mathrm{mg} / \mathrm{L}$, bilirubin: $23 \mu \mathrm{mol} / \mathrm{L}$ and alanine transaminase (ALT) $56 \mathrm{U} / \mathrm{L}$, lactate dehydrogenase (LD): $350 \mathrm{U} / \mathrm{L}$ and haptoglobin $<0.1 \mathrm{~g} / \mathrm{L}$. Initial clinical examination revealed no lymph node enlargement, but a CT-scan showed multiple enlarged lymph nodes in the abdomen. Initially the patient was slightly confused, but after a few days in hospital he became unconscious with convulsions with a Glasgow Coma Scale score of 7. A cerebral CT detected a small sub arachnoid hemorrhage, with no need for intervention. It was revealed that the patient had fallen at home a few days earlier and suffered a trauma to the head. There were normal findings in spinal puncture. The patient slowly recovered.

Albumin corrected calcium was normal at admittance, but increased rapidly from day 7 to day 17 to a maximum of $3.47 \mathrm{mmol} / \mathrm{L}$. PTH and PTH-related peptide were normal, respectively $2.7 \mathrm{pmol} / \mathrm{L}$ and $<1.9$ pmol/L. No monoclonal component was detected and vitamin D3 was low $(<10 \mathrm{pmol} / \mathrm{l})$. The patient also became increasingly dyspneic. A CT scan found interstitial pneumonitis.

The following organs were or may have been involved: (A) The skin rash was probably part of the syndrome, even though he later was diagnosed with scabies. (B) The kidney; acute interstitial nephritis associated with an allergic reaction was the most probable cause of the acute kidney injury. However, kidney biopsy was not performed. (C) The lungs; intestinal pneumonitis, as no alternative causes were plausible. Interstitial pneumonitis is common in Dress. (D) The CNS; encephalopathy has never been described as part of DRESS. Even though DRESS cannot be excluded as the cause, it is considered to be less likely than trauma and/or uremia. Uremic encephalopathy seldom develops after a short uremic period of time. Additionally, minocycline toxicity might compromise the brain. (E) Hypercalcemia has never previously been described as part of DRESS. No other cause was found, except perhaps immobilization, even though the numbers of immobile days were rather few. The patient had no calcium/PTH abnormalities related to kidney failure previous to DRESS. (F) The liver, which is often affected in Dress, was probably not involved. The patient had normal ALT values, and Bilirubin remained normal, even though it started at 11 and raised to a maximum 23,5 $\mu \mathrm{mol} / \mathrm{l}$. (G): Lymphadenopathy; the patient had no generalized enlargement of the lymph nodes, but clusters of para-aortic and subclavicular lymph nodes were detected on CT-scans. However, some of these might have been related to the chronic Y-graft infection. (H) The thrombocytopenia slowly normalized during the hospitalization and there is uncertainty about if DRESS might affect thrombocytes. Finally, (I) anemia, which has not been reported as 
part of DRESS previously. Anemia may in general be secondary to a severe inflammatory response. On the other hand Lactate Dehydrogenase was elevated and Haptoglobin was low, indicating hemolysis, which may occur as part of an allergic reaction.

Hemodialysis was started due to further increase in serum creatinine $(680 \mu \mathrm{mol} / \mathrm{L})$ and in blood urea nitrogen (37 mmol/L), and low urine output and therapy resistant hypercalcemia. The patient was treated with systemic corticosteroids because of the severe lung, kidney and skin affection. We used Prednisolone, starting at $40 \mathrm{mg}$ each day, in the low range of the recommended for acute interstitial nephritis $(0.5-2 \mathrm{mg} / \mathrm{kg})$, due to the chronic infectious condition. The patient slowly recovered, with some relapses, and had to be hospitalized for 6 weeks, but his kidney function never recovered.

\section{Discussion}

Previous reports on DRESS have mainly been casuistic, typically including a few affected organs. In this paper we present a patient with DRESS syndrome affecting multiple organs, some of which never have been reported on previously. This report illustrates the potentially life threatening outcome of DRESS, the latency between drug exposure and disease onset, and the patient also had a prolonged course with frequent relapses despite the discontinuation of the culprit drug. The report also illustrates the difficulties in addressing causality between DRESS and different symptoms and organ involvements.

A major problem is the lack of specific diagnostic tests for DRESS syndrome. Eosinophilia is thought to be essential in addition to a history of a potentially harmful drug. The incidence of DRESS is unknown. However, a prospective study estimated annual incidence to be 0.9/100.000 (Muller et al., 2003), and a mortality rate of approximately 10\% (Cacoub et al., 2011).

Numerous terms are used to describe adverse drug reactions; Drug rash with eosinophilia and systemic symptoms, drug-induced hypersensitivity syndrome and drug hypersensitivity syndrome. The unclear nosology mirrors the large diversity in the clinical manifestations of this type of adverse drug reaction, and the lack of generally accepted diagnostic criterias. Whether DRESS may include several or most of these diagnoses or should be considered as an independent diagnose is unclear. In 2007 the regiSCAR (severe cutaneous adverse reaction) scoring system was presented as a tool to differ between the multitudes of different causes to similar looking manifestations of the skin, including DRESS (Kardaun et al., 2007). Thus the regiSCARE scoring system was uses in a comprehensive review of DRESS cases reported between 1997 and 2009. A total of 172 cases was analyzed as "no/unlikely" and "probable/definite" (Cacoub et al., 2011). They concluded that the symptoms most significantly associated with DRESS were skin eruptions, hyper eosinophilia, liver involvement, fever and 
lymphadenopathy. DRESS has also been associated with the presence and reactivation of Human Hepatitis virus type 6 and 7 and Epstein Barr virus (HHV-6, 7 and EBV) (Picard et al., 2010), but the exact causality has never been described. In a prospective study including 40 DRESS patients, Epstein Barr virus, HHV-6, and HHV-7 reactivations were found in 76\% of the cases (Picard et al., 2010). In all patients, circulating CD8+ T lymphocytes were activated, and showed elevated amounts of cutaneous homing markers. Also tumor necrosis factor- $\alpha$ and interferon- $\gamma$ were secreted at large, especially in patients with the most severe visceral involvement. The culprit drug has been shown to trigger the production of virus in the virustransformes B-lymphocytes (Picard et al., 2010; Morito et al., 2014).

In our case Minocycline induced DRESS in a profound and life threatening manner. In addition to a culprit drug, grave eosinophilia and symptom relief after drug discontinuation, we found that multiple organs were affected including organs that rarely are affected (the kidneys) and organs that never have been reported to be a part of DRESS previously; hypercalcemia, anemia and maybe, but possible not, the brain. His prior multi morbid condition and especially the fact that stopping relevant antibiotic treatment could be harmful, delayed the conclusion and contributed to his severe presentation. The patient recovered slowly with multiple relapses, and is still in need of dialysis almost a year later.

DRESS might be an underdiagnosed clinical entity and there is a wide array of different diagnosis probably describing similar pathophysiological processes, and there are still no arrays satisfying the criteria to distinguish the above diagnosis.

\section{Acknowledgments: None}

\section{References}

Cacoub P, Musette P, Descamps V, Meyer O, Speirs C, Finzi L, Roujeau JC. The DRESS syndrome: a literature review. The American journal of medicine 2011; 124: 588-597.

Husain Z, Reddy BY, Schwartz RA. DRESS syndrome: Part I. Clinical perspectives. Journal of the American Academy of Dermatology 2013a; 68: 693.e1-14; quiz 706-8.

Husain Z, Reddy BY, Schwartz RA. DRESS syndrome: Part II. Management and therapeutics. Journal of the American Academy of Dermatology 2013b; 68: 709.e1-9; quiz 18-20.

Kardaun SH, Sidoroff A, Valeyrie-Allanore L, Halevy S, Davidovici BB, Mockenhaupt M, Roujeau JC. Variability in the clinical pattern of cutaneous side-effects of drugs with systemic symptoms: does a DRESS syndrome really exist? The British journal of dermatology 2007; 156: 609-611.

Morito H, Ogawa K, Fukumoto T, Kobayashi N, Morii T, Kasai T, Nonomura A, Kishimoto T, Asada H. Increased ratio of FoxP3 + regulatory T cells/CD3+ T cells in skin lesions in drug-induced hypersensitivity syndrome/drug rash with eosinophilia and systemic symptoms. Clinical and experimental dermatology 2014; 39: 284-291. 
Muller P, Dubreil P, Mahe A, Lamaury I, Salzer B, Deloumeaux J, Strobel M. Drug Hypersensitivity Syndrome in a West-Indian population. European journal of dermatology: EJD 2003; 13: 478-481.

Picard D, Janela B, Descamps V, D'Incan M, Courville P, Jacquot S, Rogez S, Mardivirin L, Moins-Teisserenc H, Toubert A, Benichou J. Drug reaction with eosinophilia and systemic symptoms (DRESS): a multiorgan antiviral $\mathrm{T}$ cell response. Science translational medicine 2010; 2: 46ra62. 